\title{
Correlation of Exchange Bias and Angle on Applied Perturbation Field and Anti-Ferromagnetic Spins
}

\author{
Xinwen $\mathrm{Fu}^{1}$, Guixin $\mathrm{Cao}^{1,2^{*}}$, Yuze $\mathrm{Gao}^{2}$, Zhiwei $\mathrm{Wu}^{1}$, Jincang Zhang ${ }^{1}$ \\ ${ }^{1}$ Department of Physics, Shanghai University, Shanghai, China \\ ${ }^{2}$ Max-Planck-Institute for Solid State Research, Stuttgart, Germany \\ E-mail: gxcao77@utk.edu \\ Received May 6, 2011; revised July 3, 2011; accepted July 19, 2011
}

\begin{abstract}
High-resolution anisotropic magneto-resistance measurement (AMR) was used to detailed study the training effect in exchange biased $\mathrm{CoO} / \mathrm{Co}$ bi-layer. The sample was cooled to $10 \mathrm{~K}$ from room temperature in the magnetic cooling field of 4000 Oe. Then we used 1500 Oe declined perturbation field to pin the magnetization orientation of the FM layer. The perturbation field forms certain angle $\Theta$ with the cooling field direction in-plane to re-induce the untrained state. The dependence of the untrained state on the angle between the direction of perturbation field and cooling field has been investigated. The AMR results reveal that the re-induced degree of untrained state is strongly correlated to the angle $\Theta$. The exchange bias field $H_{E}$ for different $\Theta$ has been determined from the AMR results, which is in apparent agreement with the Meiklejohn-Bean model. The recover degree of untrained state is the largest when the angle is $75^{\circ}$, which is different from the traditional view point that untrained state should be the maximum when it is perpendicular. The training effect is related to the FM spin orientation, which can induce the change of the interfacial AFM spin reorientation with different angles.
\end{abstract}

Keywords: Natural Asset, Financial Value, Neural Network

\section{Introduction}

Since Meiklejohn and Bean discovered exchange bias [1] this effect has obtained considerable interesting both technologically and scientifically because of its application in spintronics [2]. Exchange bias (EB) is typically set by field cooling the anti-ferromagnetic (AFM) and ferromagnetic (FM) bi-layer through the Néel temperature and is an interfacial coupling effect, as well as often associated with training effect, enhancement of coercive and so on. The training effect mechanism is important to help understand the exchange bias [3]. Although exchange bias has been discovered for 50 years [1], comprehensive understanding of its mechanism, especially the understanding of training effect mechanism is still missing [4-7]. More recently, magnetic force microscopy, neutron diffraction and anisotropic magneto-resistance (AMR) were used to investigate the training effect $[3,8]$ in thin films [9-11]. Brems et al. reported the surprising possibility to re-induce the untrained state when training effect occurred. Size effect was also involved in the training effect phenomenon included the dependence of the training effect on AFM and FM film thickness as well as the number of hysteresis loop and the magnitude of magnetic field [10,12-14]. Hoffmann [15] pointed out the specific anisotropy of AFM layer plays a crucial role in understanding of the training effect. Other approaches with different spin configurations assume that the AFM bulk plays no role in determining the interfacial spin configuration and the magnitude of EB. [16] Experimentally, it is not apparent to detect the influence of AFM spin orientation on training effect. For $\mathrm{Co} / \mathrm{CoO}$ system, several techniques have been applied to study AFM domains [17], and applied in-plane magnetic field perpendicular to the cooling field largely restores the untrained state [3]. So, the reappearance of untrained state will promote us obtain the training effect mechanism. In this case, if the applied magnetic field forms different angle with the cooling field, the transport properties of the system will be important to reflect the restoring of the untrained state and the change of the AFM bulk spin structure. The above features prompt us to undertake a new refit for the sample-hold of physics property measurement system (PPMS), which make the perturbation field 
form $15^{\circ}, 30^{\circ}, 45^{\circ}, 60^{\circ}, 75^{\circ}$, and $90^{\circ}$, respectively, with the cooling field. We select $\mathrm{Co} / \mathrm{CoO}$ bi-layer film system as studied sample, which is particularly suitable for this study because of distinct training effect [18] and initial asymmetry of AMR hysteresis after field cooling. Our goal is to give the angle dependence of untrained state and the change of the AFM bulk spin structure by using the high-resolution-anisotropic-magneto-resistance measurement (AMR). Present results reveal that the untrained state can reappear and depends on the angles closely. The recover degree of untrained state is the largest when the angle is $75^{\circ}$, which is different from the traditional view point that untrained state should be the maximum when it is perpendicular. The training effect is related to the FM spin orientation, which can induce the change of the interfacial AFM spin reorientation with different angles. The angle dependence of the EB effect can be served as the reference layer for key devices in magnetic sensors.

\section{Experiment}

The exchange coupled $\mathrm{Co} / \mathrm{CoO}$ film samples was $\mathrm{DC}$ magnetron sputtered on $\mathrm{Si}(100)$ substrates at a total pressure of $6.3 \times 10^{-1} \mathrm{~Pa}$ with $\mathrm{O}_{2}$ to Ar ratio of 1: 9, whose structure is $\mathrm{CoO}(10 \mathrm{~nm}) / \mathrm{Co}(20 \mathrm{~nm}) / \mathrm{Si}(100)$. $\mathrm{X}$-ray diffraction measurements revealed that the crystallographic structure of the samples was good single phase. The thickness of the sample was determined by SEM 4800. AMR measurements were carried out using PPMS-9 (physical property measurement system, Q/D Inc., US) with the precision $20 \mathrm{nV}$ for voltage, and 0.2 Oe for the magnetic field.

\section{Measurement and Discussion}

The AMR measurement was performed as following procedure. Firstly, the sample was cooled to $10 \mathrm{~K}$ from room temperature in the magnetic cooling field of 4000 Oe to make training effect emerge, which is well below the temperature that the exchange bias appears. Secondly, we turn the sample hold and apply 1500 Oe declined perturbation field to re-induce the untrained state. The direction of 1500 Oe perturbation field forms several certain angles $\Theta$ with that of cooling filed in-plane. Thirdly, we measure anisotropic-magneto-resistance along the cooling field direction to re-induce the occurrence of training effect. The recovered AMR value was measured also along the cooling field as shown in Figure 1, which gives schematic measurement of AMR for different $\Theta$. All the measurements and applied magnetic field are carried out in the plane, which parallel to the membrane surface.

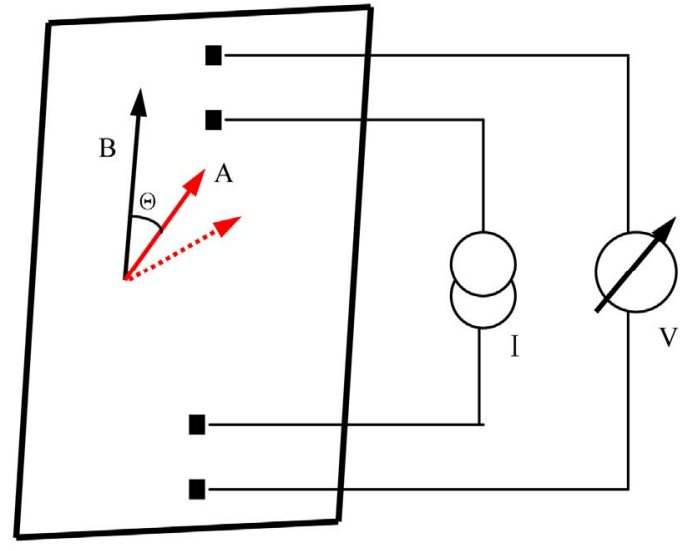

Figure 1. A schematic of the measurement of AMR for different angles, where the direction of cooling field is represented by the black arrow B, and the direction of applied external field is represented by the red arrow $A$.

The in-plane hysteresis loops in $10 \mathrm{~K}$ after the sample being cooled in the magnetic field of 4000 Oe were measured as shown in Figure 2(a). Form the figure, the distinct exchange bias and training effect can be seen. The arrows indicate the four coercive fields, respectively. Figure 2(b) gives the in-plane AMR measurement, where two subsequent hysteresis loops were measured with the field parallel to the cooling field and the current. From this figure, it can be seen that the AMR curve minimum value coincides with the magnitude of four coercive fields in the hysteresis loops as displayed by arrows, respectively, which is consistent with the result of T. Gredig et al. [11]. Since the AMR loop is corresponding to the hysteresis loop, the asymmetry of two peaks' height of AMR loop indicates the occurring degree of the training effect (untrained state) [3]. For in-plane AMR hysteresis loops (Figure 2(b)), a smaller change of resistance is observed during the first reversal at -3000 Oe when compared with the second reversal, which is dominated by domain wall motion. The magneto-resistance initially decreases slightly in the descending field branch, and then the AMR value decreases abruptly in the ascending field branch. In order to study the training effect, we used the declined perturbation field to re-induce the untrained state after training effect appears by using measurement of the second AMR loop, where the AMR peaks value decrease abruptly in the descending field branch. For the second AMR minimum, this occurs abruptly and is mainly controlled by the rotation of the magnetization [3]. The disappearance of asymmetry of two peaks' height means the occurrence of training effect. After following several AMR loops measurement, the AMR loops were the same as the second AMR loop. In general, the original AMR loop, i.e., untrained state or the recover appearance, is difficult to 
be re-induced once training effect occurs, except heating the sample through Néel temperature and then field cooling or performing an in-plane external field which form certain angle with respect to the cooling field direction [8]. This is because AFM spin orientation is difficult to be recovered to the original orientation when the AFM spin rotation occurs at the first coercive field. So, the interfacial AFM spin rotation is caused by the magnetization rotation of FM layer [19].

In polycrystalline $\mathrm{CoO} / \mathrm{Co}$ films, the resistance changes as a function of the angle $(\beta)$ between the current direction and magnetization as following [3],

$$
R(\beta) \equiv R_{0}+\Delta R \cos ^{2} \beta
$$

where, $R_{0}$ is the isotropic resistance and $\Delta R$ is the difference in resistance with the magnetization parallel and perpendicular to the current direction, $\beta$ is the angle be-

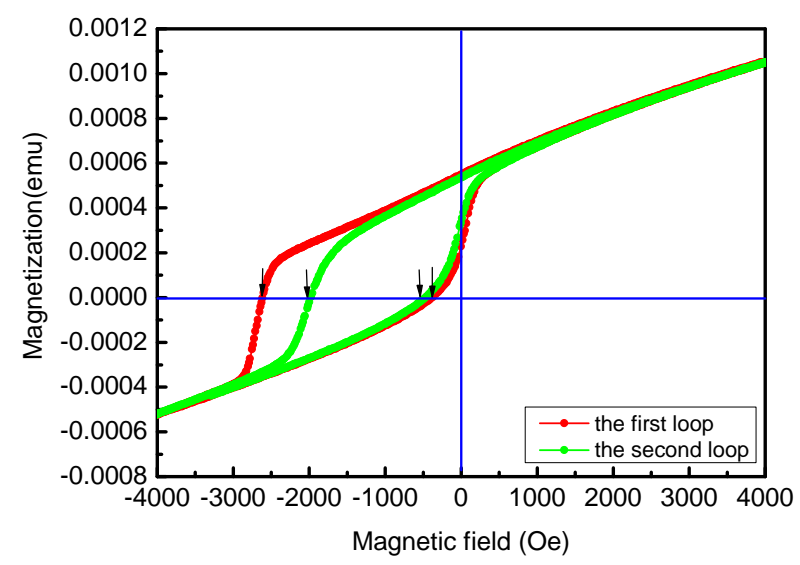

(a) tween the current and magnetization. As a result, AMR will have sensitivity to changing in the direction of magnetization by $\beta$. In order to study the re-induced degree of untrained state to understand the training effect mechanism, we detail measure the AMR value and reveal that the reversal of the training effect depends on the angle between the direction of cooling field and $1500 \mathrm{Oe}$ perturbation field. The sample is cooled from room temperature to $10 \mathrm{~K}$ in the field of $4000 \mathrm{Oe}$ in-plane. After two AMR loops measurements to induce training effect along the cooling field direction, subsequently, the AMR loop was measured after performing 1500 Oe perturbation field which forms $15^{\circ}, 30^{\circ}, 45^{\circ}, 60^{\circ}, 75^{\circ}$, and $90^{\circ}$ angle with respect to cooling field direction, respectively. The following AMR loops were measured along the field cooling direction as shown in Figure 3, which give the remarkable new results of normalized resistance after

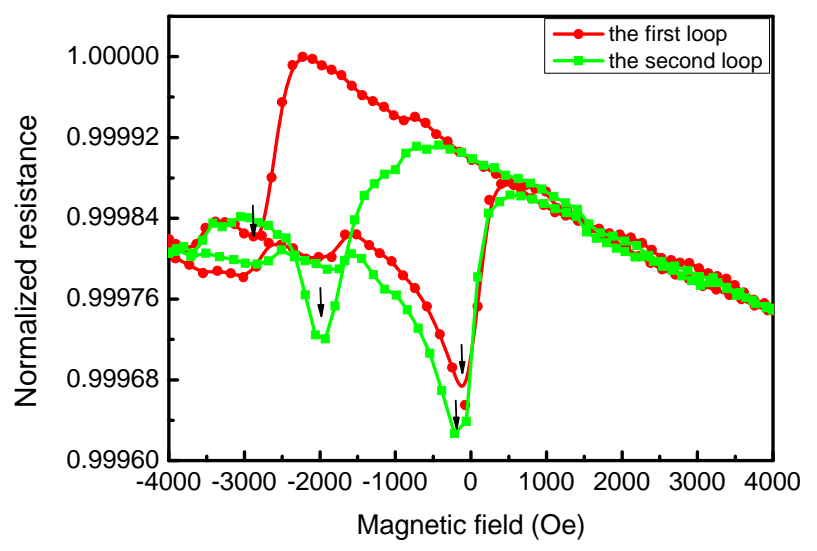

(b)

Figure 2. The hysteresis loops in $10 \mathrm{~K}$ (a) after the sample was cooled in the field of 4000 Oe. The normalized AMR loops was also measured in $10 \mathrm{~K}$. The arrows indicate that the coercivity field in is corresponding with the normalized AMR peaks value, respectively.

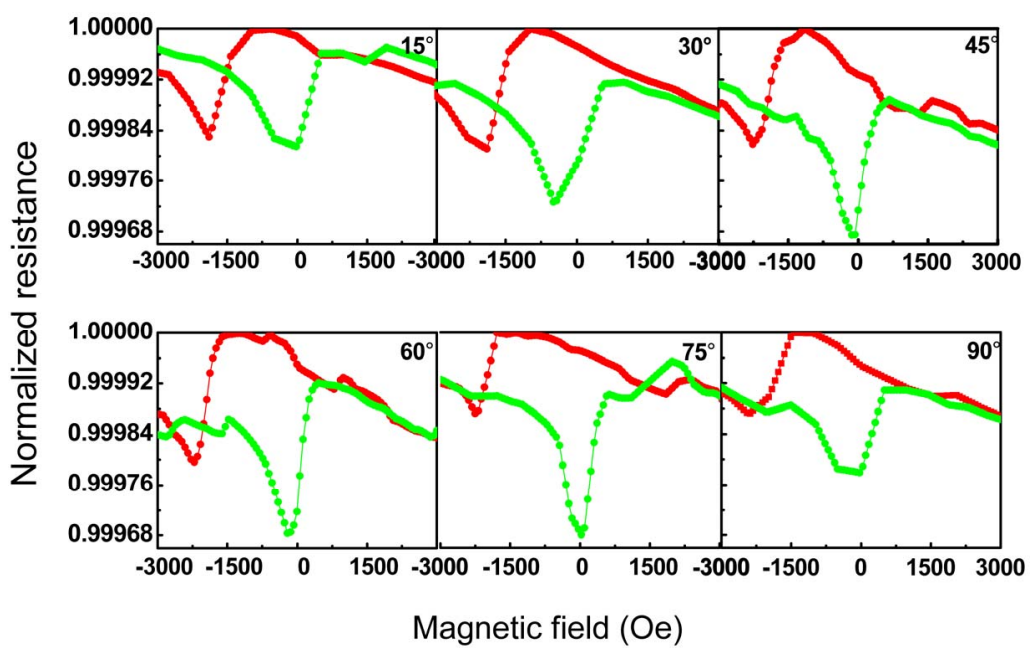

Figure 3. The recover degree of normalized AMR peaks value along the cooling field after performing a external perturbation field which forms certain angle $\Theta$ with respect to cooling field direction, $\Theta$ is $15^{\circ}, 30^{\circ}, 45^{\circ}, 60^{\circ}, 7^{\circ}$, and $90^{\circ}$, respectively. 
applied $15^{\circ}, 30^{\circ}, 45^{\circ}, 60^{\circ}, 75^{\circ}$, and $90^{\circ}$ perturbation field with respect to cooling field direction. The 1500 Oe perturbation field is large enough to change the FM layer magnetization along its orientation due to soft FM layer magnetization. In this case, however, AFM interfacial spin direction will also be changed, which deviates away from field cooling direction approximately $21^{\circ}$ after training effect occurred [3].

Since AMR curve minimum value coincides with the magnitude of four coercive fields in the hysteresis loops, we can get the exchange bias field $H_{E}$ as a function of angle $\Theta$ according to the curves of Figure 3. For the magnitude of the exchange bias $H_{E}$, the first theoretical model was proposed by Meiklejohn and Bean (MB model) [1,20], which didn't include the AFM layer thickness $t_{A F}$. Taking into account the effect of finite magneto-crystalline anisotropy of the AFM layer, Binek et al. [21] generalized the MB model and derived the following equation,

$$
H_{E}=H_{E}^{\infty}\left(1-\frac{(\Delta E)^{2}}{8 k_{A F}^{2} t_{A F}^{2}}\right)
$$

where $\Delta E=J S_{F} S_{A F}$ is interface energy per unit area and $\mathrm{S}_{\mathrm{AF} / \mathrm{F}}$ is spin of $\mathrm{AFM} / \mathrm{FM}$ ions at the interface, $J$ is their exchange coupling constant. $K_{A F}$ is the anti-ferromagnetic anisotropy constant. The above Equation (2) is obtained when magnetization of AFM layer $M_{A F}=0$ and the angle of cooling field and perturbation field $\Theta=0$. As a matter of fact, because of the spiraling spin structure in the AFM layer [22], $\Theta$ should be $g t_{A F}$ (here, $g$ is a constant). In our present $\mathrm{Co} / \mathrm{CoO}$ system, $t_{A F}$ is $10 \mathrm{~nm}$ and $\Theta$ is the formed angle between cooling field and perturbation field. Based on these, the following equation can be obtained according to original MB model,

$$
H_{E}=H_{E}^{\infty}\left(1-\frac{(\Delta E)^{2} g^{2}}{8 k_{A F}^{2} \Theta^{2}}\right)
$$

According to the assumption made in above MB approach Equation (3), when we take

$\Delta E=H_{F}^{\infty} M_{F} t_{F} \approx 10 \mathrm{erg} / \mathrm{cm}^{3}[23]$, estimate $g \approx 1.91^{\circ} / \mathrm{nm}$, and take $K_{A F} \approx 5.0^{*} 10^{6} \mathrm{erg} / \mathrm{cm}^{3}$ [24], the $H_{E}$ changing with angle $\Theta$ can be obtained as displayed in Figure 4. From the figure, it can be seen that the experiment results of $H_{E}$ nearly accords with the results of MB model. In the high-angle range, i.e., above $30^{\circ}$, the result is very in line with the MB model. There is a little deviation between experimental and theoretical data when the angle is small. We think this due to the angle smaller than anti-ferromagnetic deviation. The maximum deviation angle of anti-ferromagnetic interfacial spin is approximately $21^{\circ}$. So, the coupling FM/AFM spins follow the perturbation field rotation within approximately $21^{\circ}[3]$.

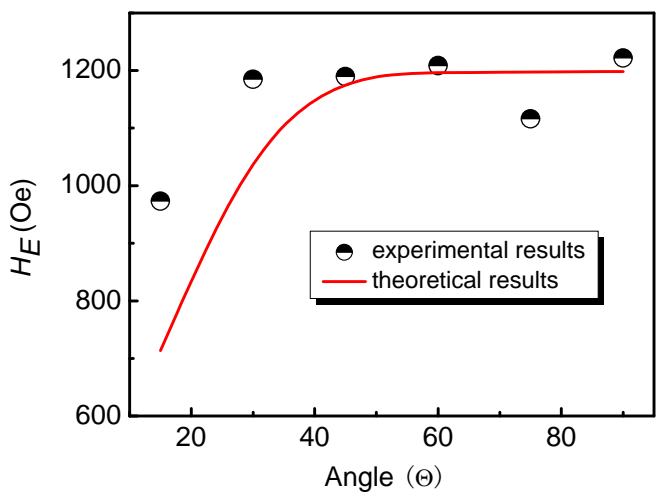

Figure 4. Exchange bias $H_{E}$ as a function of $\Theta$ for Co (20 $\mathrm{nm}) / \mathrm{CoO}(10 \mathrm{~nm})$ bi-layer at $10 \mathrm{~K}$. Solid line shows the theoretical calculation $H_{E}(\Theta)$ according to Equation (3).

However, the formula for calculating exchange bias assumed that the pinning AFM spins direction remains unchanged. So, there is negligible for the difference.

Though 1500 Oe perturbation field is not too large to change the AFM spin direction, FM magnetization rotation will change the interfacial AFM spin align. Figure 3 shows that the recovery is partially re-induced, the peak of AMR increases in the descending field branch compared with the second AMR loop. We introduce the recover degree $W$ of AMR to analyzed the re-induced untrained state for different angle $\Theta$. The recover degree of AMR is defined as the difference in height of the AMR peaks at both the descending branch of second and third AMR loops divided by the change of AMR at both the descending branch of original and second loops [3]. The value of recover degree is corresponding to the value of re-induced untrained state, i.e., the larger recover degree of asymmetry means that the untrained state is more easy to be re-induced. Figure 5 displays the calculated value of recover degree as a function of $\Theta$ according to the

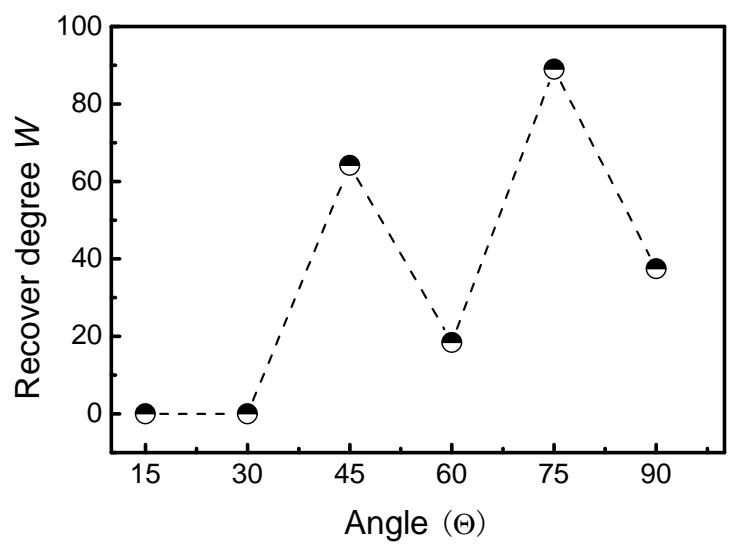

Figure 5. The recover degree $W$ of normalized AMR dependent on the angle which formed with respect to cooling field direction. The horizontal axis corresponds to the angle $\Theta$ between external field and cooling field. 
AMR results in Figure 3. From the figure, it can be seen that untrained state is partially re-induced and the reappearance of untrained state depends on the angle $\Theta$, which reflects the changing of FM layer magnetization orientation. Untrained state can be re-induced while direction of perturbation field and filed cooling form $45^{\circ}$ angle. The recover degree of untrained state is the largest while the angle is $75^{\circ}$. It's different from our traditional view point that perpendicular is the maximum.

For a qualitative understanding of the recovery and disappearance of training effect, we introduce a simple model modified by Hou et al. [25] within the framework of the Fulcomer and Charap model [26]. In this approach, many small AFM spin grains interact with ferromagnetic spin domain, and meanwhile, the FM spins was pinned by AFM spins. Our results due to the AFM spins pinning includes two aspects: one is the stable uncompensated AFM spins; the other is the couple interact between AFM spins and FM spins. They will follow FM spins rotation. After training effect occurs, the totally average AFM spins deviates away from field cooling direction, and the ferromagnetic region form a domain. We applies perturbation field 1500 Oe to change the FM spins, the part of AFM spins is also rotated with FM spins because FM spins and AFM spins couple with each other. And meanwhile, the ferromagnetic domains will also be broken as the angle increases. This makes the larger recovery with the angle variation. So, there is no recovery after training effect appearance while the angle between external field and field cooling direction is smaller than $30^{\circ}$ due to the maximum deviation angle of anti-ferromagnetic interfacial spin is approximately $21^{\circ}$ [3]. However, when the angle is greater than $30^{\circ}$, there is emergent untrained state. This is because the perturbation field breaks the ferromagnetic domains. Simultaneously, the angle is also greater than deviation angle of the antiferromagnetic spins. So, the recovery becomes distinct when the angle is large than $30^{\circ}$. However, the degree of recovery is large as the angle are $45^{\circ}$ and $75^{\circ}$, while the angle are $60^{\circ}$ and $90^{\circ}$ is relatively small. We believe that maybe caused by anisotropic of ferromagnetic. When the angle is $45^{\circ}$ and $75^{\circ}$, the ferromagnetic domain is destructed into very many smaller domains. While the angle is $60^{\circ}$ and $90^{\circ}$, the ferromagnetic domain is destructed into fewer large domains. The larger magnetic domain made exchange bias small. So, the recover degree of untrained state is the large when the angle is $45^{\circ}$ and $75^{\circ}$. For the traditional view, untrained state should be the maximum when it is perpendicular. However, they do not like us to do so detailed work, the angle we chose is so small. This issue is also needs further detailed study.

\section{Conclusions}

In summary, AMR is used to study the training effect in exchange biased $\mathrm{CoO} / \mathrm{Co}$ bi-layer. The 1500 Oe perturbation field is used to re-induce the untrained state, which forms different angle $\Theta$ with respect to cooling field direction. The dependence of the untrained state on the angle between the direction of perturbation field and cooling field reveals that the re-induced degree of untrained state is strongly correlated to the angle $\Theta$. The exchange bias field $H_{E}$ for different $\Theta$ accords with the Meiklejohn-Bean model. The recover degree of untrained state is the largest when the angle is $75^{\circ}$, which indicates that the training effect is related to the FM spin orientation and induces the change of the interfacial AFM spin reorientation with different angles. When the angle is greater than $30^{\circ}$, there is emergent untrained state. This is because the perturbation field breaks the ferromagnetic domains. Simultaneously, the angle is also greater than deviation angle of the anti-ferromagnetic spins. So, the recovery becomes distinct when the angle is large than $30^{\circ}$. However, the degree of recovery is large as the angle are $45^{\circ}$ and $75^{\circ}$, while the angle are $60^{\circ}$ and $90^{\circ}$ is relatively small. We believe that maybe caused by anisotropic of ferromagnetic. When the angle is $45^{\circ}$ and $75^{\circ}$, the ferromagnetic domain is destructed into very many smaller domains. While the angle is $60^{\circ}$ and $90^{\circ}$, the ferromagnetic domain is destructed into fewer large domains.

\section{Acknowledgements}

This work is supported by the National Natural Science Foundation of China (No. 10804068 and No. 10774097).

\section{References}

[1] W. H. Meiklejohn and C. P. Bean, "New Magnetic Anisotropy," Physical Review, Vol. 105, No. 3, 1957, pp. 904-913. doi:10.1103/PhysRev.105.904

[2] B. Dieny, V. S. Speriosu, S. S. P. Parkin, B. A. Gurney, D. R. Wilhoit and D. Mauri, "Giant Magnetoresistive in Soft Ferromagnetic Multilayers," Physical Review B, Vol. 43, No. 1, 1991, pp. 1297-1300. doi:10.1103/PhysRevB.43.1297

[3] S. Brems, K. Temst and C. V. Haesendonck, "Origin of the Training Effect and Asymmetry of the Magnetization in Polycrystalline Exchange Bias System," Physical Review Letters, Vol. 99, 2007, pp. 067201(4).

[4] J. Nogués and I. K. Schuller,"Exchange Bias," Journal of Magnetism and Magnetic Materials, Vol. 192, No. 2, 1999, pp. 203-232. doi:10.1016/S0304-8853(98)00266-2

[5] A. E. Berkowitz and K. Takano, "Exchange Anisotropy -A Review," Journal of Magnetism and Magnetic Ma- 
terials, Vol. 200, No. 1, 1999, pp. 552-570. doi:10.1016/S0304-8853(99)00453-9

[6] R. L. Stamps, "Mechanisms for Exchange Bias," Journal of Physics D, Vol. 33, 2000, pp. R247-268. doi:10.1088/0022-3727/33/23/201

[7] M. Kiwi, "Exchange Bias Theory," Journal of Magnetism and Magnetic Materials, Vol. 234, No. 3, 2001, pp. 584-595. doi:10.1016/S0304-8853(01)00421-8

[8] S. Brems, D. Buntinx, K. Temst, C. V. Haesendonck, F. Radu and H. Zabel, "Reversing the Training Effect in Exchange Biasd CoO/Co Blayers," Physical Review Letters, Vol. 95, 2005, pp. 157202(4).

[9] S. Brems, A. Volodin, C. V. Haesendonck and K. Temst, "Magnetic Force Microscopy Study of the Training Effect in Polycrystalline $\mathrm{Co} / \mathrm{CoO}$ Bilayers," Journal of Applied Physics, Vol. 103, 2008, pp. 113912(4).

[10] T. Hauet, J. A. Borchers, Ph. Mangin, Y. Henry and S. Mangin, "Training Effect in an Exchange Bias System: The role of Interfacial Domain Walls," Physical Review Letters, Vol. 96, 2006, pp. 067207(4).

[11] T. Gredig, L. N. Krivorotov and E. Dan. Dahlberg, "Magnetization Reversal in Exchange Biased $\mathrm{Co} / \mathrm{CoO}$ Probed with Anisotropic Magnetoresistance," Journal of Applied Physics, Vol. 91, 2002, pp. 7760-7763. doi:10.1063/1.1447181

[12] S. Polisetty, S. Sahoo and C. Binek, "Scaling Behavior of the Exchange-Bias Training Effect," Physical Review B, 76, 2007, pp. 184423(9).

[13] K. Zhang, T. Zhao and H. Fujiwara, "Training Effect of Exchange Biased Iron-Oxide/Ferromagnet Systems," Journal of Applied Physics, Vol. 89, No. 11, 2001, pp. 6910-6912. doi:10.1063/1.1360682

[14] P. Y. Yang, C. Song, F. Zeng and F. Pan, "Tuning the Training Effect in Exchange Biased NiO/Ni Bilayers," Applied Physics Letters, Vol. 92, 2008, pp. 243113(3).

[15] A. Hoffmann, "Symmetry Driven Irreversibilities at Ferromagnetic-Antiferromagnetic Interfaces," Physical Review Letters, Vol. 93, 2004, pp. 097203(4).

[16] M. Kiwi, J. Mejía-López, R. D. Portugal and R. Ramírez, "Exchange-Bias Systems with Compensated Interfaces," Applied Physics Letters, Vol. 75, 1999, pp. 3995-3997. doi:10.1063/1.125517

[17] C. L. Chien, V. S. Gornakov, V. I. Nikitenko, A. J. Shapiro and R. D. Shull, "Hybrid Domain Walls and Antiferromagnetic Domains in Exchange-Coupled Ferromagnet/Antiferromagnet Bilayers," Physical Review B, Vol. 68, 2003, pp. 014418(5).

[18] M. Gruyters and D. Riegel, "Strong Exchange Bias by a Single Layer of Independent Antiferromagnetic Grains: The CoO/Co Model System," Physical Review B, Vol. 63, 2000, pp. 052401(4).

[19] T. Hauet, S. Mangin, J. McCord, F. Montaigne and E. E. Fullerton, "Exchange-Bias Training Effect in TbFe/GdFe: Micromagnetic Mechanism," Physical Review B, Vol. 76, 2007, pp. 144423(5).

[20] W. H. Meiklejohn, "Exchange Anisotropy-A Review," Journal of Applied Physics, Vol. 33, 1962, pp. 1328-1335. doi:10.1063/1.1728716

[21] Ch. Binek, A. Hochstrat, and W. Kleemann, "Exchange Bias in a Generalized Meiklejohn-Bean Approach," Journal of Magnetism and Magnetic Materials, Vol. 234, 2001, pp. 354-358.

[22] F. Y. Yang, and C. L. Chien, "Spiraling Spin Structure in an Exchange-Coupled Antiferromagnetic Layer," Physical Review Letters, Vol. 85, No. 12, 2000, pp. 2597-2600. doi:10.1103/PhysRevLett.85.2597

[23] A. L. Kobrinskii, A. M. Goldman, M. Varela and S. J. Pennycook, "Thickness Dependence of the Exchange Bias in Epitaxial Manganite Bilayers," Physical Review B, Vol. 79, 2009, pp. 094405(7).

[24] S. Chikazumi, "Physics of Ferromagnetism," Oxford University Press, New York, 1997, p. 329.

[25] C. Hou, H. Fujiwara and F. Ueda, "Effect of F/AF Coupling Strength on the Magnetization Process of F/AF System," Journal of Magnetism and Magnetic Materials, Vol. 198, No. 1-3, 1999, pp. 450-452. doi:10.1016/S0304-8853(98)01165-2

[26] E. Fulcomer and S. H. Charap, "Thermal Fluctuation Aftereffect Model for Some Systems with FerromagneticAntiferromagnetic Coupling," Journal of Applied Physics, Vol. 43, 1972, pp. 4190-4199. doi:10.1063/1.1660894 